\title{
Role of imitation and limited rehabilitation capacity on the spread of drug abuse
}

\author{
Josiah Mushanyu*
}

\begin{abstract}
Objectives: We formulate a mathematical model for the spread of drug abuse using non linear ordinary differential equations. The model seeks to investigate both peer influence and limited rehabilitation effects on the dynamics of drug abuse. Peer-influence is modelled through the mechanism of imitation and limited rehabilitation is described using a special treatment function. Center manifold theory is used to show that the model exhibits the phenomenon of backward bifurcation. Matlab has been used to carry out numerical simulations to support theoretical findings.

Results: The model analysis shows that the model has multiple equilibria. It has been shown that the classical $\mathcal{R}_{a}$ - threshold is not the key to control drug abuse within a population. In fact drug abuse problems may persist in the population even with subthreshold values of $\mathcal{R}_{a}$. This was shown to result, in particular when, $\omega, \eta_{1}$ and $\eta_{2}$ are high enough such that $\omega>\omega^{*}, \eta_{1}>\eta_{1}^{*}$ and $\eta_{2}>\eta_{2}^{*}$. The results suggest the need for comprehensive and accessible substance abuse treatment services to curtail drug abuse.
\end{abstract}

Keywords: Drug abuse, Imitation, Reproduction number, Rehabilitation capacity

\section{Introduction}

Drug abuse has increased in recent years and is now an epidemic globally. The magnitude of the world drug problem becomes more apparent when considering that more than 1 out of 10 drug users is a problem drug user and the vast majority of these individuals continue to have no access to treatment [1]. There continues to be a large "treatment gap" for substance abuse problems as many countries have a large shortfall in the provision of services. According to the United Nations Office on Drugs and Crime [1], only one out of every six problem drug users in the world has access to treatment. Generally, the number of patients in need of rehabilitation often exceeds the carrying capacities of drug treatment facilities, especially those funded by the state.

Several mathematical models describing the spread of psycho-social ills in a community have been proposed, see for example, drug epidemics [2-9], alcoholism [1016], smoking [17-19]. The basic assumption in most drug abuse models is that there is a direct proportional

\footnotetext{
*Correspondence: mushanyuj@gmail.com

Department of Mathematics, University of Zimbabwe, Box MP 167, Mount

Pleasant, Harare, Zimbabwe
}

relationship between the number of drug users in need of treatment and the available health care resources present. In this paper, we develop a mathematical model that takes into account the possibility of the number of drug abusers in need of rehabilitation exceeding the capacity of rehabilitation centers. Recruitment into rehabilitation (inpatient or outpatient) is denoted by $H(U)$ and defined as follows:

$$
H(U)=\frac{\alpha U}{1+\omega U}
$$

where $U$ represents the proportion of individuals abusing drugs, $\alpha$ is the maximum rehabilitation uptake per unit of time and $\omega$ measures the extent of the effect of the problem of demand for treatment. Firstly, observe that for small $U, H(U) \approx \alpha U$, that is, when the number of drug users is not too large, then the rate of entering treatment is proportional to the number of drug users present. Secondly, observe that for large $U, H(U) \approx \alpha / \omega$, this means that the rate of entering rehabilitation takes a maximum value $\alpha / \omega$. Finally, when $\omega=0$, we again obtain the result as in the first case, $H(U)=\alpha U$, that is, the function returns to a linear function mostly used in previous 
drug abuse models. Amongst drug abusers who are seeking help through rehabilitation, we have that a proportion $p$ of these individuals are recruited into inpatient rehabilitation and the complementary proportion $(1-p)$ are recruited into outpatient rehabilitation. It is also important to note that epidemic models including treatment functions of the form (1) are found in [20-23].

We also include peer influence effects on the spread of drug abuse by assuming that the recruitment process happens through the mechanism of imitation. In this paper, we use the recruitment function given in [11]. Compared to previous drug epidemic models [2-9], a key novelty of our model is the inclusion of both imitation and limited rehabilitation on the dynamics of drug abuse.

The paper is arranged as follows; in "Model formulation" section, we formulate and establish the basic properties of the model. The model is analysed for stability in "Model analysis" section. In "Numerical simulations" section, we carry out some numerical simulations. Parameter estimation is also presented in this section. The paper is concluded in the "Conclusions" section.

\section{Main text \\ Model formulation}

The model divides the population into four classes, $S(t)$, $U(t), R_{o p}(t)$ and $R_{i p}(t)$. The class $S(t)$ represents the population at risk of being initiated into drug abuse. The class $U(t)$ denotes those abusing drugs, $R_{o p}(t)$ denotes those in rehabilitation as out-patients and $R_{i p}(t)$ denotes those in rehabilitation as in-patients. The total local population is thus given by

$$
N(t)=S(t)+U(t)+R_{o p}(t)+R_{i p}(t) .
$$

The general population enter the susceptible population at a rate $\Lambda$, that is, the demographic process of individuals reaching age 15 in the modelling time period. Susceptible individuals become drug users upon contact with individuals in compartments $U$ or $R_{o p}$. This results from the assumption that those in inpatient rehabilitation do not have contact with the population at risk. The per capita contact rate $\beta_{1}$ is a product of the effective number of contacts $c_{1}$, between drug users not in rehabilitation and the susceptible population, and the probability $\hat{\beta}_{1}$, that a contact results into initiation into drug use, that is, $\beta_{1}=c_{1} \hat{\beta}_{1}$. The per capita contact rate $\beta_{2}$ is a product of the effective number of contacts $c_{2}$, between drug users in outpatient rehabilitation and the susceptible population, and the probability $\hat{\beta}_{2}$, that a contact results into initiation into drug use, that is, $\beta_{2}=c_{2} \hat{\beta}_{2}$. Individuals under outpatient rehabilitation quit drug abuse permanently at a rate $\delta_{1}$ and individuals under inpatient rehabilitation quit drug abuse permanently at a rate $\delta_{2}$. The general population experience natural death at a rate $\mu$.
Drug users undergoing outpatient rehabilitation relapse into drug use at a rate $\rho_{1}$ whereas those undergoing inpatient rehabilitation relapse at a rate $\rho_{2}$. The relapse is thus assumed to be a voluntary process, that is not influenced by interaction with users. We allow the transfer from outpatient to inpatient rehabilitation, this happens at a rate $\gamma_{1}$. We also allow the transfer from inpatient to outpatient rehabilitation, this rate is represented by $\gamma_{2}$. We assume that individuals in each compartment are indistinguishable and there is homogeneous mixing. We have the following general set of nonlinear ordinary differential equations:

$$
\left\{\begin{array}{l}
\frac{d S}{d t}=\Lambda-f\left(S, U, R_{o p}\right)-\mu S \\
\frac{d U}{d t}=f\left(S, U, R_{o p}\right)+\rho_{1} R_{o p}+\rho_{2} R_{i p}-\mu U-\frac{\alpha U}{1+\omega U} \\
\frac{d R_{o p}}{d t}=\gamma_{2} R_{i p}-\left(\mu+\gamma_{1}+\rho_{1}+\delta_{1}\right) R_{o p}+\frac{(1-p) \alpha U}{1+\omega U} \\
\frac{d R_{i p}}{d t}=\gamma_{1} R_{o p}-\left(\mu+\gamma_{2}+\rho_{2}+\delta_{2}\right) R_{i p}+\frac{p \alpha U}{1+\omega U},
\end{array}\right.
$$

with the initial conditions:

$$
\begin{aligned}
S(0) & =S_{0}>0, U(0)=U_{0} \geq 0, R_{o p}(0) \\
& =R_{o p 0} \geq 0, R_{i p}(0)=R_{i p 0} \geq 0,
\end{aligned}
$$

where

$$
\begin{aligned}
f\left(S, U, R_{o p}\right) & =\beta_{1} S U\left(1+\eta_{1} U\right)+\beta_{2} S R_{o p}\left(1+\eta_{2} R_{o p}\right) \\
& =\beta_{1}\left(S U\left(1+\eta_{1} U\right)+\theta S R_{o p}\left(1+\eta_{2} R_{o p}\right)\right) .
\end{aligned}
$$

Here $\beta_{2}=\theta \beta_{1}$, with $\theta=1$ signifying that the chance of initiating drug abuse habit upon contact with an individual in $U$ or $R_{o p}$ is the same, $\theta \in(0,1)$ signifying a reduced chance of initiating drug abuse habit upon contact with an individual in $R_{o p}$ as compared to an individual in $U$, $\theta>1$ signifies an increased rate of initiating drug abuse habit upon contact with an individual in $R_{o p}$ as compared to an individual in $U$.

\section{Model analysis \\ Model properties}

Invariant region It follows from system (2) that

$$
\frac{d N}{d t} \leq \Lambda-\mu\left(S+U+R_{o p}+R_{i p}\right) .
$$

Then, $\limsup _{t \rightarrow \infty} N \leq \frac{\Lambda}{\mu}$. Thus, the feasible region for system (2) is

$$
\Omega=\left\{\left(S, U, R_{o p}, R_{i p}\right) \in \mathbb{R}_{+}^{4} \mid N \leq \frac{\Lambda}{\mu}\right\} .
$$


It is easy to verify that the region $\Omega$ is positively invariant with respect to system (2), see for instance [3-5].

\section{The drug-free equilibrium and the abuse reproduction} number

Model system (2) always has a drug-free equilibrium $\mathcal{D}_{0}=\left(\frac{\Lambda}{\mu}, 0,0,0\right)$. Denote the abuse reproduction number of model system (2) by

$$
\begin{aligned}
\mathcal{R}_{a} & =\mathcal{R}_{U}+\mathcal{R}_{R_{o p}} \quad \text { where } \\
\mathcal{R}_{U} & =\left(\frac{\Lambda}{\mu}\right)\left[\frac{\beta_{1}\left(1-\Phi_{1}\right)}{\mu\left(1-\Phi_{1}\right)+\alpha p\left(1-\Phi_{2}\right)+\alpha(1-p)\left(1-\Phi_{3}\right)}\right] \text { and } \\
\mathcal{R}_{R_{o p}} & =\left(\frac{\Lambda}{\mu h_{1} h_{2}}\right)\left[\frac{\beta_{2}\left((1-p) \alpha h_{2}+p \alpha \gamma_{2}\right)}{\mu\left(1-\Phi_{1}\right)+\alpha p\left(1-\Phi_{2}\right)+\alpha(1-p)\left(1-\Phi_{3}\right)}\right]
\end{aligned}
$$

where $h_{1}$ and $h_{2}$ are defined as before and $g_{1}=\frac{\Lambda}{\mu} \beta_{1}-(\mu+\alpha), g_{2}=\frac{\Lambda}{\mu} \beta_{2}+\rho_{1}$. The local stability of the drug-free equilibrium is determined by the following submatrix of $J\left(\mathcal{D}_{0}\right)$,

$$
\bar{J}\left(\mathcal{D}_{0}\right)=\left[\begin{array}{ccc}
g_{1} & g_{2} & \rho_{2} \\
(1-p) \alpha & -h_{1} & \gamma_{2} \\
p \alpha & \gamma_{1} & -h_{2}
\end{array}\right]
$$

with

$$
\begin{aligned}
\Phi_{1} & =\frac{\gamma_{1} \gamma_{2}}{h_{1} h_{2}}, \Phi_{2}=\frac{\gamma_{1} \gamma_{2}+\gamma_{2} \rho_{1}+\rho_{2} h_{1}}{h_{1} h_{2}}, \\
\Phi_{3} & =\frac{\gamma_{1} \gamma_{2}+\gamma_{1} \rho_{2}+\rho_{1} h_{2}}{h_{1} h_{2}}, \\
h_{1} & =\mu+\gamma_{1}+\rho_{1}+\delta_{1} \text { and } h_{2}=\mu+\gamma_{2}+\rho_{2}+\delta_{2} .
\end{aligned}
$$

We can clearly note that $\gamma_{1} \gamma_{2} \leq h_{1} h_{2}$ and so $\left(1-\Gamma_{1}\right) \geq 0$. Also, $\quad \gamma_{1} \gamma_{2}+\gamma_{2} \rho_{1}+\rho_{2} h_{1} \leq h_{1} h_{2} \quad$ and $\gamma_{1} \gamma_{2}+\gamma_{1} \rho_{2}+\rho_{1} h_{2} \leq h_{1} h_{2}$. Therefore, $\mathcal{R}_{a}$ is non-negative. The abuse reproduction number $\mathcal{R}_{a}$ of the model, is the average number of secondary cases generated by one drug user during his/her duration of drug use in a population of completely potential drug users.

\section{Local stability of the drug-free steady state}

Theorem 1 The drug-free equilibrium $\mathcal{D}_{0}$ is locally asymptotically stable when $\mathcal{R}_{a}<1$ and is unstable when $\mathcal{R}_{a}>1$.

Proof The Jacobian matrix of model system Eq. (2) at $\mathcal{D}_{0}$ is given by

$$
J\left(\mathcal{D}_{0}\right)=\left[\begin{array}{cccc}
-\mu & -\frac{\Lambda}{\mu} \beta_{1} & \frac{\Lambda}{\mu} \beta_{2} & 0 \\
0 & g_{1} & g_{2} & \rho_{2} \\
0 & (1-p) \alpha & -h_{1} & \gamma_{2} \\
0 & p \alpha & \gamma_{1} & -h_{2}
\end{array}\right]
$$

Since all off-diagonal elements are positive, we now consider matrix $-\bar{J}\left(\mathcal{D}_{0}\right)$. We claim that $-\bar{J}\left(\mathcal{D}_{0}\right)$ is an $M-$ matrix. Multiplying matrix $-\bar{J}\left(\mathcal{D}_{0}\right)$ by the positive $3 \times 1$ matrix

$$
W_{1}=\left[\begin{array}{c}
h_{1} h_{2}\left(1-\Phi_{1}\right) \\
p \alpha \gamma_{2}+(1-p) \alpha h_{2} \\
(1-p) \alpha \gamma_{1}+p \alpha h_{1}
\end{array}\right]
$$

we have

$$
-\bar{J}\left(\mathcal{D}_{0}\right) \cdot W_{1}=\left(1-\mathcal{R}_{a}\right) \cdot W_{2}
$$

where $W_{2}$ is a positive $3 \times 1$ matrix given by $W_{2}=\left[\begin{array}{c}h_{1} h_{2}\left[\mu\left(1-\Phi_{1}\right)+\alpha p\left(1-\Phi_{2}\right)+\alpha(1-p)\left(1-\Phi_{3}\right)\right] \\ 0 \\ 0\end{array}\right]$.

Then, it follows from $M$-matrix theory that all eigenvalues of $\bar{J}\left(\mathcal{D}_{0}\right)$ have negative real parts, which implies the local asymptotic stability of the drug-free equilibrium if $\mathcal{R}_{a}<1$. On the other hand, it can be shown that the determinant of $\bar{J}\left(\mathcal{D}_{0}\right)$ is given by

$$
\begin{aligned}
\operatorname{det} \bar{J}\left(\mathcal{D}_{0}\right)= & h_{1} h_{2}\left[\mu\left(1-\Phi_{1}\right)+\alpha p\left(1-\Phi_{2}\right)\right. \\
& \left.+\alpha(1-p)\left(1-\Phi_{3}\right)\right]\left(\mathcal{R}_{a}-1\right) .
\end{aligned}
$$

Thus, if $\mathcal{R}_{a}<1$, then matrix $\bar{J}\left(\mathcal{D}_{0}\right)$ has eigenvalues with negative real parts, which implies the stability of the drug-free equilibrium. This completes the proof. 


\section{The drug-persistent equilibrium point}

The drug-persistent equilibrium $\mathcal{D}^{*}=\left(S^{*}, U^{*}, R_{o p}^{*}, R_{i p}^{*}\right)$ always satisfies

$$
\left\{\begin{array}{l}
\Lambda-f\left(S^{*}, U^{*}, R_{o p}^{*}\right)-\mu S^{*}=0, \\
f\left(S^{*}, U^{*}, R_{o p}^{*}\right)+\rho_{1} R_{o p}^{*}+\rho_{2} R_{i p}^{*}-\mu U^{*}-\frac{\alpha U^{*}}{1+\omega U^{*}}=0, \\
\gamma_{2} R_{i p}^{*}-\left(\mu+\gamma_{1}+\rho_{1}+\delta_{1}\right) R_{o p}^{*}+\frac{(1-p) \alpha U^{*}}{1+\omega U^{*}}=0, \\
\gamma_{1} R_{o p}^{*}-\left(\mu+\gamma_{2}+\rho_{2}+\delta_{2}\right) R_{i p}^{*}+\frac{p \alpha U^{*}}{1+\omega U^{*}}=0 .
\end{array}\right.
$$

From the last two equations of (5) we have that

$$
R_{o p}^{*}=\frac{\Psi_{1} U^{*}}{1+\omega U^{*}} \text { and } R_{i p}^{*}=\frac{\Psi_{2} U^{*}}{1+\omega U^{*}}
$$

where

$$
\Psi_{1}=\frac{\alpha p \gamma_{2}+\alpha(1-p) h_{2}}{h_{1} h_{2}\left(1-\Phi_{1}\right)} \text { and } \Psi_{2}=\frac{\alpha p h_{1}+\alpha(1-p) \gamma_{1}}{h_{1} h_{2}\left(1-\Phi_{1}\right)} .
$$

Substituting expressions (6) into the first equation of (5), we get

$$
S^{*}=\frac{\Lambda\left(1+\omega U^{*}\right)^{2}}{\left(\mu+\beta_{1} U^{*}\left(1+\eta_{1} U^{*}\right)\right)\left(1+\omega U^{*}\right)^{2}+\beta_{2} \Psi_{1} U^{*}\left(1+\omega U^{*}+\eta_{2} \Psi_{1} U^{*}\right)} .
$$

$$
h\left(x_{1}, x_{2}, x_{3}\right)=\beta_{1}\left(x_{1} x_{2}\left(1+\eta_{1} x_{2}\right)+\theta x_{1} x_{3}\left(1+\eta_{2} x_{3}\right)\right) .
$$

Let $\beta_{1}$ be the bifurcation parameter, $\mathcal{R}_{a}=1$ corresponds to

\section{Backward bifurcation}

Conditions for the existence of backward bifurcation follow from Theorem 4.1 proven in [24]. Let us make the following change of variables:

$$
S=x_{1}, U=x_{2} R_{o p}=x_{3}, R_{i p}=x_{4} \text {, so that } \mathrm{N}=\sum_{n=1}^{4} x_{n} .
$$

We now use the vector notation $X=\left(x_{1}, x_{2}, x_{3}, x_{4}\right)^{T}$. System (2) can be written in the form $\frac{d X}{d t}=F(t, x(t))=\left(f_{1}, f_{2}, f_{3}, f_{4}\right)^{T}$, where

$$
\left\{\begin{array}{l}
x_{1}^{\prime}(t)=p \Lambda-h\left(x_{1}, x_{2}, x_{3}\right)-\mu x_{1}=f_{1}, \\
x_{2}^{\prime}(t)=h\left(x_{1}, x_{2}, x_{3}\right)+\rho_{1} x_{3}+\rho_{2} x_{4}-\mu x_{2}-\frac{\alpha x_{2}}{1+\omega x_{2}}=f_{2}, \\
x_{3}^{\prime}(t)=\gamma_{2} x_{4}-h_{1} x_{3}+\frac{\alpha(1-p) x_{2}}{1+\omega x_{2}}=f_{3} \\
x_{4}^{\prime}(t)=\gamma_{1} x_{3}-h_{2} x_{4}+\frac{\alpha p x_{2}}{1+\omega x_{2}}=f_{4}
\end{array}\right.
$$

with
Substituting expressions (6) and (7) into the second equation of (5) leads to the following sixth order polynomial equation

$$
U^{*}\left(\chi_{5} U^{* 5}+\chi_{4} U^{* 4}+\chi_{3} U^{* 3}+\chi_{2} U^{* 2}+\chi_{1} U^{*}+\chi_{0}\right)=0 .
$$

Solving (8) gives $U^{*}=0$ which corresponds to the drugfree equilibrium or

$$
\chi_{5} U^{* 5}+\chi_{4} U^{* 4}+\chi_{3} U^{* 3}+\chi_{2} U^{* 2}+\chi_{1} U^{*}+\chi_{0}=0,
$$

where the coefficients $\chi_{i}, 1 \leq i \leq 5$ are in Additional file 1: Appendix S1. We can clearly note that, $\chi_{0}>0 \Leftrightarrow \mathcal{R}_{a}<1$ and $\chi_{0}<0 \Leftrightarrow \mathcal{R}_{a}>1$. The number of possible positive real roots of the polynomial (9) can be determined using the Descartes Rule of Signs. The number of positive roots are at most five. 


$$
\begin{aligned}
w_{1}= & -h_{1} h_{2}\left(\mu\left(1-\Phi_{1}\right)+\alpha p\left(1-\Phi_{2}\right)\right. \\
& \left.+\alpha(1-p)\left(1-\Phi_{3}\right)\right), \\
w_{2}= & \mu h_{1} h_{2}\left(1-\Phi_{1}\right), w_{3}=\alpha \mu\left((1-p) h_{2}+p \gamma_{2}\right), \\
w_{4}= & \alpha \mu\left(p h_{1}+(1-p) \gamma_{1}\right) .
\end{aligned}
$$

Further, the left eigenvector of $J^{*}\left(\mathcal{D}_{0}\right)$, associated with the zero eigenvalue at $\beta_{1}=\beta_{1}^{*}$ is given by $v=\left(v_{1}, v_{2}, v_{3}, v_{4}\right)^{T}$, where

$$
\begin{aligned}
& v_{1}=0, \quad v_{2}=h_{1} h_{2}\left(1-\Phi_{1}\right)+\alpha(1-p) \theta h_{2}+\alpha p \theta \gamma_{2}, \\
& v_{3}=h_{2}\left(\theta(\alpha+\mu)+\rho_{1}\right)+\rho_{2}\left(\gamma_{1}-\alpha \theta p\right), \\
& v_{4}=\rho_{2}\left(h_{1}+\alpha \theta(1-p)\right)+\gamma_{2}\left(\theta(\alpha+\mu)+\rho_{1}\right) .
\end{aligned}
$$

The computations of $\mathbf{a}$ and $\mathbf{b}$ are necessary in order to apply Theorem 4.1 in Castillo-Chavez and Song [24]. For system (10), the associated non-zero partial derivatives of $F$ at the drug-free equilibrium are in Additional file 1: Appendix S2. It thus follows that
Note that $\omega^{*}>0, \eta_{1}^{*}>0$ and $\eta_{2}^{*}>0$. Also note that if $\omega>\omega^{*}, \eta_{1}>\eta_{1}^{*}$ and $\eta_{2}>\eta_{2}^{*}$ then $\mathbf{a}>0$ and $\mathbf{a}<0$ if $\omega<\omega^{*}, \eta_{1}<\eta_{1}^{*}$ and $\eta_{2}<\eta_{2}^{*}$. Lastly,

$$
\mathbf{b}=\Lambda\left(h_{2}\left(\alpha \theta(p-1)-h_{1}\right)+\gamma_{2}\left(\gamma_{1}-\alpha \theta p\right)\right)^{2}>0 .
$$

We thus have the following result

Theorem 2 If $\omega>\omega^{*}, \eta_{1}>\eta_{1}^{*}$ and $\eta_{2}>\eta_{2}^{*}$, then model system (2) has a backward bifurcation at $\mathcal{R}_{a}=1$.

\section{Results and discussion \\ Numerical simulations}

Parameter estimation Since we can rarely enumerate the incidence of drug users, data from treatment centers can be used as proxy for estimating parameters for drug related issues. We use data obtained from previous mathematical models with inpatient and outpatient rehabilita-

$$
\begin{aligned}
\mathbf{a}= & v_{1} w_{1} w_{2} \frac{\partial^{2} f_{1}}{\partial x_{1} \partial x_{2}}+v_{1} w_{1} w_{3} \frac{\partial^{2} f_{1}}{\partial x_{1} \partial x_{3}}+v_{1} w_{2}^{2} \frac{\partial^{2} f_{1}}{\partial x_{2}^{2}}+v_{1} w_{3}^{2} \frac{\partial^{2} f_{1}}{\partial x_{3}^{2}}+v_{2} w_{1} w_{2} \frac{\partial^{2} f_{2}}{\partial x_{1} \partial x_{2}} \\
& +v_{2} w_{1} w_{3} \frac{\partial^{2} f_{2}}{\partial x_{1} \partial x_{3}}+v_{2} w_{2}^{2} \frac{\partial^{2} f_{2}}{\partial x_{2}^{2}}+v_{2} w_{3}^{2} \frac{\partial^{2} f_{2}}{\partial x_{3}^{2}}+v_{3} w_{2}^{2} \frac{\partial^{2} f_{3}}{\partial x_{2}^{2}}+v_{4} w_{2}^{2} \frac{\partial^{2} f_{4}}{\partial x_{2}^{2}} \\
= & 2 \alpha \omega v_{2} w_{2}^{2}-2(1-p) \alpha \omega v_{3} w_{2}^{2}-2 \alpha p \omega v_{4} w_{2}^{2}+\beta_{1}^{*} v_{2} w_{1} w_{2}+\theta \beta_{1}^{*} v_{2} w_{1} w_{3} \\
& +\frac{2 \Lambda \beta_{1}^{*} \eta_{1} v_{2} w_{2}^{2}}{\mu}+\frac{2 \theta \Lambda \beta_{1}^{*} \eta_{2} v_{2} w_{3}^{2}}{\mu} \\
= & {\left[A \omega-\mu^{2} h_{1} h_{2}\left(1-\Phi_{1}\right) v_{2}^{2} \beta_{1}^{*}\right]+\left[B \eta_{1}-\mu \alpha p h_{1} h_{2}\left(1-\Phi_{2}\right) v_{2}^{2} \beta_{1}^{*}\right] } \\
& +\left[C \eta_{2}-\mu \alpha(1-p) h_{1} h_{2}\left(1-\Phi_{3}\right) v_{2}^{2} \beta_{1}^{*}\right] \\
= & A\left(\omega-\omega^{*}\right)+B\left(\eta_{1}-\eta_{1}^{*}\right)+C\left(\eta_{2}-\eta_{2}^{*}\right),
\end{aligned}
$$

where

$$
\begin{aligned}
\omega^{*} & =\frac{\mu^{2} h_{1} h_{2}\left(1-\Phi_{1}\right) v_{2}^{2} \beta_{1}^{*}}{A}, \\
\eta_{1}^{*} & =\frac{\mu \alpha p h_{1} h_{2}\left(1-\Phi_{2}\right) v_{2}^{2} \beta_{1}^{*}}{B}, \\
\eta_{2}^{*} & =\frac{\mu \alpha(1-p) h_{1} h_{2}\left(1-\Phi_{3}\right) v_{2}^{2} \beta_{1}^{*}}{C},
\end{aligned}
$$

with

$$
\begin{aligned}
A= & 2 \alpha \mu^{2} h_{1}^{2} h_{2}^{2}\left(1-\Phi_{1}\right)^{2} \times\left[\left((1-\theta) \mu+\delta_{1}\right)\left((1-p) \rho_{2}+\gamma_{2}\right)\right. \\
& \left.+\left(\mu+\delta_{2}\right)\left((1-\theta) \mu+\mu \theta p+\gamma_{1}+\delta_{1}+p \rho_{1}\right)\right], \\
B= & 2 \Lambda \mu h_{1}^{2} h_{2}^{2}\left(1-\Phi_{1}\right)^{2} v_{2} \beta_{1}^{*} \text { and } \\
C= & 2 \Lambda \mu \theta \alpha^{2}\left((1-p) h_{2}+p \gamma_{2}\right)^{2} v_{2} \beta_{1}^{*} .
\end{aligned}
$$

tion $[4,5]$. Some of the parameter values will be obtained from literature.

Parameter values used for numerical simulations are given in Table 1.

Numerical results We carry out detailed numerical simulations using matlab to support our theoretical findings. The initial conditions used are: $S(0)=0.95, U(0)=0.05$, $R_{o p}(0)=0, R_{i p}(0)=0$.

Figures 1 and 2 illustrate the effect of varying parameters $\omega$ and $\eta_{1}$ on the prevalence of drug abuse. Figures 1 and 2 demonstrate that increasing $\omega$ and $\eta_{1}$ results in an increase in the prevalence of drug abuse. This is a reflection that limited rehabilitation and imitation are of major concern in the fight against drug abuse. 
Table 1 Parameter values used in numerical simulations

\begin{tabular}{llll}
\hline Parameter & Range & Value & Source \\
\hline$\beta_{1}$ & $0.10-0.21$ & 0.105 & {$[7]$} \\
$\beta_{2}$ & $0-0.10$ & 0.063 & {$[6]$} \\
$\omega$ & $0-1$ & 0.62 & {$[5]$} \\
$\alpha$ & $0-0.05024$ & 0.02827 & {$[4]$} \\
$p$ & $0-1$ & 0.352 & {$[4]$} \\
$\eta_{1}$ & $0-1$ & 0.24 & Assumed \\
$\eta_{2}$ & $0-1$ & 0.13 & Assumed \\
$\delta_{1}$ & $0.001-1$ & 0.01 & {$[4]$} \\
$\delta_{2}$ & $0.01-1$ & 0.3142 & {$[4]$} \\
$\rho_{1}$ & $0-0.054$ & 0.0382 & {$[4]$} \\
$\rho_{2}$ & $0-0.0235$ & 0.0020 & {$[4]$} \\
$\gamma_{1}$ & $0-0.06012$ & 0.02961 & {$[4]$} \\
$\gamma_{2}$ & $0-0.008$ & 0.003 & {$[4]$} \\
$\Lambda$ & $0.028-0.080$ & 0.04 & {$[7]$} \\
$\mu$ & $0.019-0.021$ & 0.020 & {$[25]$} \\
\hline
\end{tabular}

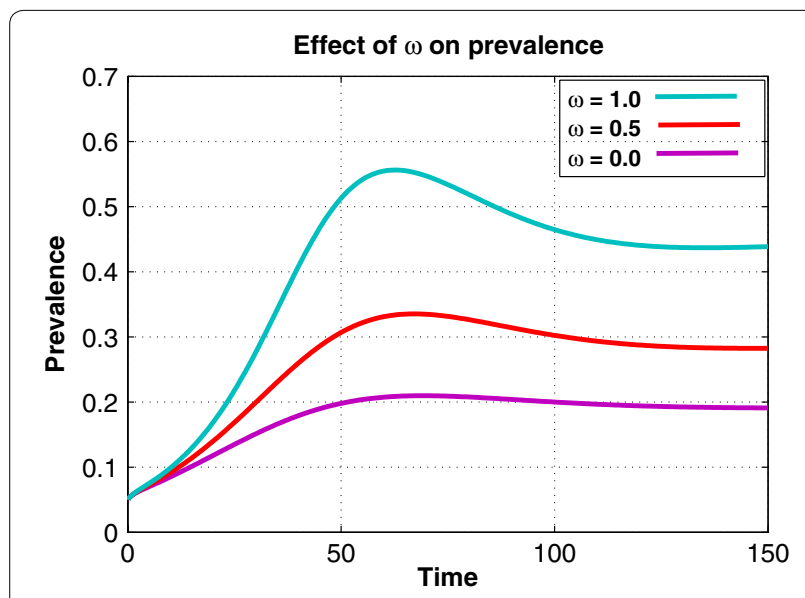

Fig. 1 Effects of varying $\omega$ on the prevalence of drug abuse, starting from 0 up to 1.0 with a step size of 0.5

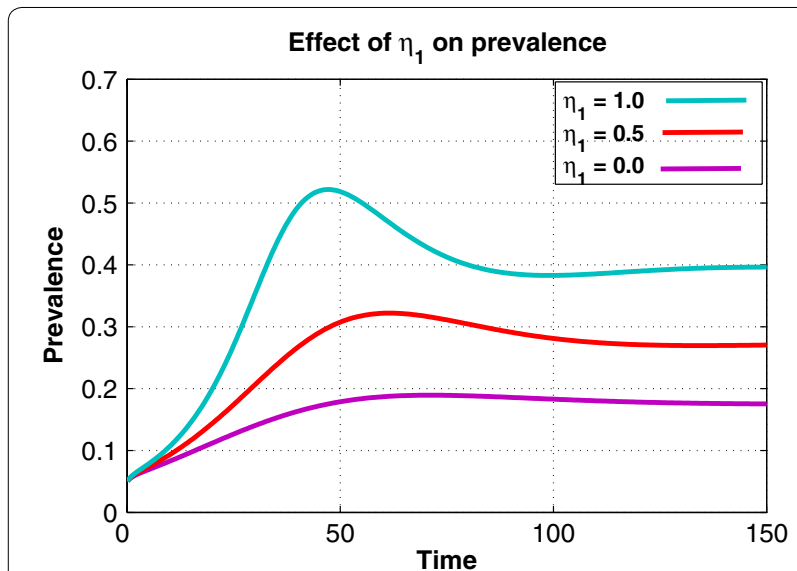

Fig. 2 Effects of varying $\eta_{1}$ on the prevalence of drug abuse, starting from 0 up to 1.0 with a step size of 0.5

\section{Conclusions}

A mathematical model that incorporates imitation and limited rehabilitation has been formulated using nonlinear ordinary differential equations. It has been shown that the classical $\mathcal{R}_{a}$-threshold is not the key to control drug abuse within a population. In fact drug abuse problems may persist in the population even with subthreshold values of $\mathcal{R}_{a}$. This was shown to result, in particular when $\omega, \eta_{1}$ and $\eta_{2}$ are high enough such that $\omega>\omega^{*}, \eta_{1}>\eta_{1}^{*}$ and $\eta_{2}>\eta_{2}^{*}$. Considerable effort should be directed towards reducing $\omega, \eta_{1}$ and $\eta_{2}$, this done by increasing the value of $\omega^{*}, \eta_{1}^{*}$ and $\eta_{2}^{*}$ so as to avoid backward bifurcation. Also, results from the model application show that increasing $\omega$ and $\eta_{1}$ lead to an increase in the prevalence of drug abuse. Thus, communities should have suitable capacity for the treatment of drug abusers and specific health and/or education programs may be employed to reduce the imitation coefficient $\eta_{1}$.

\section{Limitations}

Like in any model development, the model is not without limitations.

- The model did not take into account contextual dynamics, such as drug supply chains or changes in interdiction.

- Also, the study presented here ignored detailed social and economic characteristics.

- Other initiation processes, not included in this work, for instance, initiation by self-conversion, drug supply chains etc. may form part of the author's future research considerations.

\section{Additional file}

Additional file 1. Appendices S1, S2

\section{Authors' contributions}

The author read and approved the final manuscript.

\section{Acknowledgments}

The author acknowledges, with thanks, the support of the Department of Mathematics, University of Zimbabwe for the production of this manuscript.

\section{Competing interests}

The author declares no competing interests.

\section{Availability of data and materials}

Estimation of parameters have been stated throughout the body of the paper and included in the reference section. The graphs were produced using the MATLAB software that is available from https://www.mathworks.com/produ cts/matlab.html.

Consent to publish

Not applicable. 
Ethics approval and consent to participate

No ethical approval was required for this project as this is secondary research.

\section{Funding}

Not applicable.

\section{Publisher's Note}

Springer Nature remains neutral with regard to jurisdictional claims in published maps and institutional affiliations.

Received: 13 February 2018 Accepted: 6 July 2018

Published online: 18 July 2018

\section{References}

1. United Nations Office on Drugs and Crime. World drug report. New York: United Nations publication; 2015

2. Mulone G, Straughan B. A note on heroin epidemics. Math Biosci. 2009;208:138-41.

3. Mushanyu J, Nyabadza F, Muchatibaya G, Stewart AGR. Modelling multiple relapses in drug epidemics. Ricerche di Matematica. 2015. https://doi. org/10.1007/s11587-015-0241-0.

4. Mushanyu J, Nyabadza F, Stewart AGR. Modelling the trends of inpatient and outpatient rehabilitation for methamphetamine in the Western Cape province of South Africa. BMC Res Notes. 2015b;8:797.

5. Mushanyu J, Nyabadza F, Muchatibaya G, Stewart AGR. Modelling drug abuse epidemics in the presence of limited rehabilitation capacity. Bull Math Biol. 2016. https://doi.org/10.1007/s11538-016-0218-5.

6. Njagarah JBH, Nyabadza F. Modelling the impact of rehabilitation, amelioration and relapse on the prevalence of drug epidemics. J Biol Syst. 2013;21:1350001.

7. Nyabadza F, Hove-Musekwa SD. From heroin epidemics to methamphetamine epidemics: modelling substance abuse in a South African province. Math Biosci. 2010:225:132-40.

8. Nyabadza F, Njagarah JBH, Smith RJ. Modelling the dynamics of crystal meth (Tik) abuse in the presence of drug-supply shains in South Africa. Bull Math Biol. 2012. https://doi.org/10.1007/s11538-012-9790-5.

9. White $\mathrm{E}$, Comiskey C. Heroin epidemics, treatment and ODE modelling. Math Biosci. 2007:208:312-24
10. Benedict B. Modeling alcoholism as a contagious disease: how infected drinking buddies spread problem drinking. SIAM News. 2007;40(3):11-3.

11. Buonomo B, Lacitignola D. Modeling peer influence effects on the spread of highrisk alcohol consumption behavior. Ricerche di Matematica. 2013:63:101-17.

12. Mubayi A, Greenwood PE, Castillo-Chavez C, Gruenewald PJ, Gorman DM The impact of relative residence times on the distribution of heavy drinkers in highly distinct environments. Socio Econ Plan Sci. 2010;44:4556.

13. Mulone G, Straughan B. Modelling binge drinking. Int J Biomath. 2012; 5:1250005.

14. Sánchez F, Wang X, Castillo-Chavez C, Gorman DM, Gruenewald PJ. Drinking as an epidemic: a simple mathematical model with recovery and relapse. In: Alan Marlatt G, Witkiewitz K, editors. Therapists guide to evidence-based relapse prevention. New York: Academic Press; 2007.

15. Sharma S, Samanta GP. Analysis of a drinking epidemic model. Int J Dyn Control. 2015. https://doi.org/10.1007/s40435-015-0151-8.

16. Walters CE, Straughan B, Kendal JR. Modelling alcohol problems: total recovery. Ricerche Matematica. 2013:62:33-53.

17. Alkhudhari Z, Al-Sheikh S, Al-Tuwairqi S. Global dynamics of a mathematical model on smoking. Int Sch Res Not. 2014. https://doi. org/10.1155/2014/847075.

18. Bissell JJ, Caiado CCS, Goldstein M, Straughan B. Compartmental modelling of social dynamics with generalised peer incidence. Math Models Methods Appl Sci. 2014;24:719-50.

19. Sharomi $O$, Gumel $A B$. Curtailing smoking dynamics: a mathematical modeling approach. Appl Math Comput. 2008;195:475499.

20. Hu Z, Ma W, Ruan S. Analysis of SIR epidemic models with nonlinear incidence rate and treatment. Math Biosci. 2012;238:12-20.

21. Zhang J, Jia J, Song X. Analysis of an SEIR epidemic model with saturated incidence and saturated treatment function. Sci World J. 2014. https:// doi.org/10.1155/2014/910421.

22. Zhang X, Liu XN. Backward bifurcation of an epidemic model with saturated treatment function. J Math Anal Appl. 2008;348:433443.

23. Zhou L, Fan M. Dynamics of an SIR epidemic model with limited medical resources revisited. Nonlin Anal Real World Appl. 2012;13:312-24.

24. Castillo-Chavez C, Song B. Dynamical models of tuberclosis and their applications. Math Biosci Eng. 2004;1(2):361-404.

25. Jamison DT, Feachmen RG, Makgoba MW, Bos ER, Baingana FK, Hofman KJ, Rogo KO. Disease and mortality in sub-saharan Africa. 2nd ed. Washington DC: World Bank; 2006.
Ready to submit your research? Choose BMC and benefit from:

- fast, convenient online submission

- thorough peer review by experienced researchers in your field

- rapid publication on acceptance

- support for research data, including large and complex data types

- gold Open Access which fosters wider collaboration and increased citations

- maximum visibility for your research: over $100 \mathrm{M}$ website views per year

At $\mathrm{BMC}$, research is always in progress.

Learn more biomedcentral.com/submissions 